\begin{tabular}{|c|l|}
\hline Title & Effects of growth on residual stress distribution al ong the radial depth of cortical cylinders from bovine femurs. \\
\hline Author(s) & Y amada, Satoshi; Tadano, Shigeru \\
\hline Citation & $\begin{array}{l}\text { Journal of biomechanics, 46(13), 2130-2136 } \\
\text { https://doi.org/10.1016/.jbiomech.2013.07.002 }\end{array}$ \\
\hline Issue Date & 2013-09-03 \\
\hline Doc URL & http://hdl.handle.net/2115/53104 \\
\hline Type & article (author version) \\
\hline File Information & HUSCA PO90413.pdf \\
\hline
\end{tabular}

Instructions for use 


\title{
Effects of Growth on Residual Stress Distribution along the Radial Depth of Cortical Cylinders from Bovine Femurs
}

\author{
Satoshi YAMADA ${ }^{\text {a }}$, Shigeru TADANO ${ }^{\text {a,* }}$
}

a Division of Human Mechanical Systems and Design, Faculty of Engineering, Hokkaido University, N13 W8, Kita-ku, Sapporo, Hokkaido 060-8628, Japan

*Corresponding Author:

Shigeru TADANO, $\mathrm{PhD}$

Professor, Division of Human Mechanical Systems and Design, Faculty of Engineering, Hokkaido University

N13 W8, Kita-ku, Sapporo, Hokkaido 060-8628, JAPAN

Tel/Fax: +81(JAPAN)-11-706-6405

E-mail: tadano@eng.hokudai.ac.jp

Word count: 3405 words (Introduction through Discussion)

Type of manuscript: Original article

Keywords: Bone, Biomechanics, X-ray Diffraction, Growth Effect, Residual Stress 


\section{Abstract}

Residual stress is defined as the stress that remains in bone tissue without any

external forces. This study investigated the effects of growth on residual stress

distributions from the surface to deeper regions of cortical cylinders obtained from less-than-one-month-old (Group Y) and two-year-old (Group M) bovine femurs. In these experiments, five diaphysis specimens from each group were used. Residual stress was measured using a high-energy synchrotron white X-ray beam to penetrate X-rays into the deeper region of the bone specimens. The measurements in the cortical cylinders from Groups $\mathrm{Y}$ and $\mathrm{M}$ were performed at 0.5 - and 1-mm intervals, respectively, from the outer surface to the deeper region of the diaphysis specimens at four positions: anterior, posterior, lateral, and medial. The residual stress was calculated on the basis of variation in the interplanar spacing of hydroxyapatite crystals in the bone tissue. According to the results, the diaphysis specimens from Group Y were not subjected to large residual stresses (average -1.2 $\mathrm{MPa}$ and 2.4 $\mathrm{MPa}$ at the surface region and $1.5 \mathrm{~mm}$ depth, respectively). In Group $\mathrm{M}$, the surface region of the diaphysis specimens was subjected to tensile residual stresses (average 6.7 $\mathrm{MPa}$ ) and the deeper 
region was subjected to compressive stresses (average $-8.2 \mathrm{MPa}$ at $3 \mathrm{~mm}$ depth). There was a strong significant difference between both these regions. The value of residual stresses at the surface region of the diaphysis specimens in both the groups had a positive statistical correlation with the cortical thickness at the measured locations. (247 words) 


\section{Introduction}

Stress/strain state in bone tissue is essential for understanding the bone strength and bone adaptation. Researches attempted to obtain in vivo measurements of bone strain under external loads by invasive procedures with strain gauges glued to the bone surface (Al Nazer et al., 2012). X-ray diffraction is an alternative promising tool to obtain in vivo measurements of the stress/strain in bone tissue because X-rays have nondestructive and noninvasive properties (Tadano and Giri, 2011). Some studies reported the deformation of hydroxyapatite (HAp) crystals in bone tissue under external loads using X-ray diffraction (e.g., Almer and Stock, 2005; Fujisaki et al., 2006; Gupta et al., 2006; Fujisaki and Tadano, 2007; Almer and Stock, 2007; Tadano et al., 2008; Akhtar et al., 2011; Giri et al., 2012; Yamada et al., 2013).

The presence of residual stress in bone tissue, which is defined as the stress that remains in the tissue without any external forces, has been examined using X-ray diffraction techniques (Tadano and Okoshi, 2006; Giri et al., 2008; Yamada and Tadano, 2010; Yamada et al., 2011a; Yamada et al., 2011b). In general, residual stress is one of the significant factors for the strength of materials. On bone tissue, the magnitude of 
residual stresses along the bone axis at the bone surface of rabbit extremities correlated with the osteon population density (Yamada et al., 2011a). The residual stress may be related to the bone formation and reconstruction, and may be a circumstantial finding of the adapted state of the bone. However, the distribution, generation mechanism, and the biomechanical implications of the residual stress in bone tissue are not understood. To profoundly enhance the understanding of generation mechanisms and biomechanical implications of residual stress in bone tissue, it is important to study the effects of growth on residual stresses.

The authors have proposed the $\sin ^{2} \psi$ method of X-ray diffraction to measure residual stress in bone tissue (Yamada and Tadano, 2010; Yamada et al., 2011a). In the previous studies, residual stresses at the diaphysis surfaces of limb bones were measured using characteristic Mo-K $\alpha$ X-rays generated by an X-ray tube. However, the residual stresses in the deeper region of the diaphysis of limb bones could not be measured using this method. To understand the generation mechanisms of residual stress in bone tissue, it is quite important to investigate the residual stress distribution from the surface to the deeper region of the diaphysis. The authors have proposed an 
alternative method for measurement of residual stresses in deeper regions of the diaphysis using synchrotron white X-rays (Yamada et al., 2011b). The high-energy synchrotron white X-rays can pass through thick bone specimens.

The aim of the current study was to investigate the effects of growth on residual stress distributions along the radial direction from the surface to the deeper region of the diaphysis from bovine femurs of less-than-one-month-old and two-year-old animals using synchrotron white X-rays.

\section{Materials and methods}

\subsection{Synchrotron measurement method}

A coordinate system is fixed at each measurement location as shown in Fig. 1.

The $x, y$, and $z$ axes correspond to the bone axis, circumferential, and radial directions, respectively. The angle of inclination, $\psi$, is defined as the angle between the $z$-axis and the normal direction of the diffracted lattice planes of HAp crystals.

The interplanar spacing $d$ of a specific lattice plane in the HAp crystals is uniform in the nonstrained state, but varies with the direction under the stress state. 
When the cortical bone is locally stretched in the bone axis owing to tensile residual stress, the $d$ is largest in the $\psi=90^{\circ}$ direction, and is smallest in the $\psi=0^{\circ}$ direction, as suggested in Fig. 1. The relationship between $d$ and $\psi$ depends on the magnitude of the residual stress. Hence, the macroscopic residual stress can be estimated on the basis of the $d-\psi$ relationship.

In this study, the relationship between bone tissue stress $\sigma^{B}$ and lattice strain of HAp crystals, $\varepsilon^{H}$, in the cortical bone is assumed to be described by Eq. (1).

$$
\left(\begin{array}{c}
\varepsilon_{x}^{H} \\
\varepsilon_{y}^{H} \\
\varepsilon_{z}^{H}
\end{array}\right)=\left[\begin{array}{ccc}
\frac{1}{E^{*}} & -\frac{v^{*}}{E^{*}} & -\frac{v^{*}}{E^{*}} \\
-\frac{v^{*}}{E^{*}} & \frac{1}{E^{*}} & -\frac{v^{*}}{E^{*}} \\
-\frac{v^{*}}{E^{*}} & -\frac{v^{*}}{E^{*}} & \frac{1}{E^{*}}
\end{array}\right]\left(\begin{array}{c}
\sigma_{x}^{B} \\
\sigma_{y}^{B} \\
\sigma_{z}^{B}
\end{array}\right)
$$

The $E^{*}$ and $v^{*}$ are X-ray elastic constant and X-ray Poisson's ratio, respectively, and these are the elastic properties between the bone tissue stress and the HAp crystal strain. The $x, y$, and $z$ axes were defined as the principal axes and it was assumed that the diaphysis was not subject to residual stress in the radial direction $\left(\sigma^{B}{ }_{z}=0\right)$. 
In this study, the energy-dispersive X-ray diffraction with synchrotron white X-rays was used to measure the $d-\psi$ relationship. The relationship between the wavelength $\lambda$ and energy $W$ of X-rays is expressed as in Eq. (2), where $h$ is Planck's constant and $c$ is the speed of light.

$$
\lambda=\frac{h c}{W}
$$

Bragg's law, the fundamental equation for X-ray diffraction, is expressed as in Eq. (3)

$$
2 d \sin \theta=n \lambda
$$

where $\theta$ and $d$ are the Bragg angle and the interplanar spacing at a specific lattice plane ( $h k l$ ) of HAp crystals, respectively. Using Eq. (3), we express the relationship between $d$ and $W$ as in Eq. (4). 


$$
d=\frac{h c}{2 W \sin \theta}
$$

The $d$ can be measured from the energy $W$ of the diffracted X-rays when $\theta$ is fixed. Here, the strain in the HAp lattice plane, $\varepsilon^{H}$, is defined as in Eq. (5)

$$
\varepsilon^{H}=\frac{d-d_{0}}{d_{0}}
$$

where $d_{0}$ is the interplanar spacing of the nonstrained state. It is possible to measure the HAp crystal strain $\varepsilon^{H}{ }_{\psi}$ for an angle of inclination, $\psi$, as shown in Fig. 1, and $\varepsilon^{H}{ }_{\psi}$ is described in terms of $\varepsilon^{H}{ }_{x}$ and $\varepsilon^{H}{ }_{z}$ as in Eq. (6).

$$
\varepsilon_{\psi}^{H}=\varepsilon_{x}^{H} \sin ^{2} \psi+\varepsilon_{z}^{H}\left(1-\sin ^{2} \psi\right)
$$

Equation (6) can be expressed as Eq. (7) using Eq. (1). 


$$
\varepsilon_{\psi}^{H}=\frac{1+v^{*}}{E^{*}} \sigma_{x}^{B} \sin ^{2} \psi-\frac{v^{*}}{E^{*}}\left(\sigma_{x}^{B}+\sigma_{y}^{B}\right)
$$

Partial differentiation of Eq. (7) with respect to $\sin ^{2} \psi$ gives the following expression.

$$
\frac{E^{*}}{1+v^{*}} \frac{\partial \varepsilon_{\psi}^{H}}{\partial\left(\sin ^{2} \psi\right)}=\sigma_{x}^{B}
$$

Therefore, the residual stress $\sigma_{x}^{B}$ in the bone axis can be calculated using the relationship between interplanar spacing $d_{\psi}$ and angle of inclination, $\psi$, as in Eq. (9)

$$
\sigma_{x}^{B}=\frac{E^{*}}{d_{0}\left(1+v^{*}\right)} \frac{\partial d_{\psi}}{\partial\left(\sin ^{2} \psi\right)} \equiv K_{d} \frac{\partial d_{\psi}}{\partial\left(\sin ^{2} \psi\right)},
$$

where $K_{d}$ is the stress constant with a value of $21000 \mathrm{MPa} / \mathrm{nm}$ (Yamada et al., 2011b) and the $K_{d}$ value was measured using a four-point bending test of a bone specimen with X-ray irradiation.

\subsection{Specimens}


Five femurs were obtained from less-than-one-month-old (Group Y) and two-year-old (23.2 \pm 1.5 months old, Group M) bovines, respectively. The specimens were frozen at $-35^{\circ} \mathrm{C}$ until further preparation. The mid-diaphysis part of the femur was used for the study. The specimens were cut using a slow-speed diamond wheel saw (Model 650: South Bay Technology Inc., USA) such that the measurement position was at both the center of the femur and the center of the diaphysis specimen (Fig. 2). The length of the femurs from Group $\mathrm{M}$ was $450 \mathrm{~mm}$ and the diaphysis specimen lengths were $60 \mathrm{~mm}$ along the longitudinal axis of the bone. In Group Y, the average length of femurs was $235 \pm 14 \mathrm{~mm}$. The bone marrow and the soft tissue around the surfaces were removed and the specimens were air-dried at room temperature. To fix the specimens for X-ray diffraction measurement, one end was bonded to an acrylic plate using epoxy resin.

\subsection{Measurements of residual stress}

As shown in Fig. 2, the diaphysis specimens from Group Y were measured at 0.5-mm intervals from the outer surface to the deeper region of the specimens at four 
positions: anterior, posterior, lateral, and medial. The Group M samples were measured at 1-mm intervals. In the study, anterior was defined as the head (cranial) side and posterior was defined as the tail (caudal) side of the bovine when the limbs were extended.

The synchrotron white X-ray diffraction was performed at the BL28B2 beamline of SPring-8, a large synchrotron radiation facility managed by RIKEN and JASRI. As shown in Fig. 3, the diaphysis specimens were irradiated with a synchrotron white X-ray beam, where the incident angle $\theta$ was $2.5^{\circ}$. The intensity of the synchrotron white X-rays was optimized with an aluminum absorber and collimated using a slit (Slit 1). Scattered X-rays were eliminated from the diffracted X-rays by two slits (Slits 2 and 3 ) and the diffraction angle $2 \theta$ was set to $5^{\circ}$. The measured region of residual stress at each location was a parallelepiped of approximately $5500 \times 250 \times 240 \mu \mathrm{m}$. The longitudinal directions of the measured regions were aligned along the circumferential directions of the diaphysis specimens. The diffracted X-rays were measured using a solid-state detector (SSD) and the pulse height distribution of the X-rays was analyzed using a multichannel analyzer (MCA; 4096 channels). To calibrate the MCA, 
fluorescent X-rays of $\mathrm{Pb}-\mathrm{K} \alpha_{1}$ and $\mathrm{Sn}-\mathrm{K} \alpha_{1}$ were measured.

Figure 4 shows the typical X-ray diffraction profile of a diaphysis specimen.

The horizontal axis indicates the energy of diffracted X-rays. The peak of the (002)

plane in the HAp crystals was sharp with a distinct maximum intensity. Its position in the spectrum was determined as the midpoint of the full width at two-thirds maximum intensity of the profile (FWTTM method) using the energy range between 39.8 and 43.1 $\mathrm{keV}$ to calculate the interplanar spacing $d_{\psi}$. This spacing was measured at the four angles of inclination $(\psi): 90.0^{\circ}, 71.6^{\circ}, 63.4^{\circ}$, and $56.8^{\circ}$, and $\partial d_{\psi} / \partial\left(\sin ^{2} \psi\right)$ in Eq. (9) was calculated using the linear least-squares method.

\section{Results}

Figure 5 shows the typical cross-sectional shapes at the measurement positions of the diaphysis specimens for each group, and Table 1 lists the specimen size and shape of each cross section. The cross-sectional area of the diaphyses from Group $M$ were four times the size of those from Group $\mathrm{Y}$, and the cortical thickness and diaphysis width were also almost doubled. The diaphyses from Group Y were more 
circular than those from Group $\mathrm{M}$ and the cortical thickness in the anterior part was significantly larger in Group M.

Figure 6 shows typical distributions of residual stress in the bone axis from the outer surface region to the deeper region of diaphysis specimens from (a) Group Y and (b) Group $\mathrm{M}$ at the four positions. The horizontal axis indicates the depth from the surface of the diaphysis specimen at each position. The deepest locations indicated in Fig. 6 do not correspond to the inner surfaces of the diaphysis specimens, because X-ray profiles could not be obtained around the inner surface regions based on the length of the X-ray pathway. In Group Y, residual stresses did not vary with the depth from the surface. The magnitude of residual stress was nominal, a minimum of $-8.5 \mathrm{MPa}$ at the medial surface position and a maximum of $8.5 \mathrm{MPa}$ at the 1-mm depth of the posterior position. Further, there was no distinctive distribution among the four positions. Conversely, a trend was observed in the specimens from Group $\mathrm{M}$ toward tensile residual stresses around the surface region and compressive residual stresses inside the diaphysis. In the anterior position, the residual stress was $23.9 \mathrm{MPa}$ at the surface region. The average of residual stress at depths of 1-7 mm was -16.3 MPa. 
Figure 7 shows the average distributions of residual stress at the (a) anterior and (b) posterior positions. Each value indicates the average of five specimens and the errors represent their standard deviations. Because the deepest measured locations were not the same in each of the five specimens, Fig. 7 indicates the distribution at the deepest location where the residual stress could be measured for all specimens. Among the anterior measurements, the residual stresses for Group Y did not vary and were near zero for both the surface and deeper regions of the diaphysis. Alternately, the residual stresses for Group $\mathrm{M}$ were tensile at the surface region and compressive in the deeper region of the diaphysis. In the posterior measurements, the similar trend was also observed in Group M: tensile stress in the surface region and compressive stresses in the deeper regions. However, the differences between the groups were smaller than those in the anterior measurements and the standard deviations were substantial.

Figure 8 shows the difference in residual stresses between the surface and the deeper regions of the diaphysis specimens and the averages of cross-sectional area. In this figure, the deeper region of Group $M$ was defined as the 3-mm depth from the surface, and that of Group Y was defined as the 1.5-mm depth. The specimens from 
Group $\mathrm{M}$ were twice the length of those from Group Y. Each bar of residual stresses indicates the average of five specimens for each of the four positions.

The Group M distribution clearly indicated that the surface region was subjected to tensile residual stresses and the deeper region was subjected to compressive stresses. There was a strong significant difference between the residual stresses measured at the surface region and in the deeper region $(p<0.001)$. Conversely, Group Y was not subjected to such large residual stresses, with measurements near zero. Furthermore, there was no significant difference between the surface and deeper regions in Group Y $(p=0.095)$.

Figure 9 shows the relationship between the residual stresses at the surface region of the diaphysis specimens and the cortical thickness at the measured locations. The value of residual stresses had a positive statistical correlation with the cortical thickness $(r=0.44, p<0.01)$.

\section{Discussion}

In the measured regions of residual stress at the surface, approximately $90 \%$ 
of the volume fitted inside the diaphysis specimens as shown in Fig. 5. The volume of measured region scarcely changed with the locations. When the specimens were rotated to change the angle $\psi$, the measured region was also rotated corresponding to approximately $80 \%$ of the original volume at each location. These changes may have less impact on the results. Further, the chemical impurities of bone mineral may affect the X-ray diffraction profile and the $d$ value. In this study, the variation in the full-width at half-maximum (FWHM) of the (002) plane peak in the profile was small in both the groups. It suggests that the impurity effects on the value of residual stresses can be negligible. Further, the same value of $K_{d}$ was used to calculate the residual stresses in both the young and mature bones in this study because the $K_{d}$ value was not different between these groups according to the measurement of a young bone specimen $(3 \times 28$ $\times 0.6 \mathrm{~mm}$ ) using the previous procedure (Yamada et al., 2011a).

The limitation of this study was the use of the air-dried specimens cut out from the whole femurs. To evaluate the effects of the cutting on the stress state, three strain gauges glued on the surface of the mid-diaphysis in a thawed whole femur of a mature bovine and the diaphysis specimen was taken from it. As a result, the strain 
changes in the bone axis were smaller than $50 \mu$ and it suggests that the cutting may have less impact on the measured residual stresses. Further, the measurement method as expressed in Fig. 1 and Eq. (9) detected only the deviatoric stress and this study focused on the distribution of that stress. Although there may be hydrostatic compressive deformation related with the sample volume changes due to the air-drying, the drying process was the same for all specimens and the effects may also have less impact on the measured residual stresses in this study. In fact, the trend that there were tensile residual stresses around the surface region of the mature bones corresponded to the results of the previous study with the limb bones of adult rabbits that kept saline solution just before the measurements (Yamada et al., 2011a). Further, there are no significant effects of prolonged cryopreservation on the mechanical properties of cortical bone (Salai et al., 2000). Hence, the sample preparation may not affect the results in the current study.

This study shows the differences in residual stress distribution between the young and mature bovine femurs. The young bones were not subjected to residual stresses, whereas larger residual stresses were observed in the mature bones. In addition, the mature bones showed a trend toward tensile residual stresses at the surface region 
and compressive residual stresses in the deeper region. The results suggest that residual stress is generated during growth, satisfying the equilibrium of forces between the surface and the deeper regions of the diaphysis.

The differences in residual stress may be related to mechanical loads applied to bone tissue in vivo during bone formation or reconstruction. The young bovine femurs have been subjected to minimal loading caused by body weight or movement as compared with the mature bovine femurs. The anterior location of the mature bovine femurs was thickest among the four locations, the difference probably caused by bone adaptation to the distribution of in vivo loading due to bending. This may explain the significant difference in residual stress distribution between the young and mature bovine femurs at the anterior location as compared to those in the posterior location, as shown in Fig. 7. In fact, the value of residual stresses at the surface region of the diaphysis in both the groups had a positive statistical correlation with the cortical thickness at the measured locations as shown in Fig. 9. These growth effects may influence the generation of residual stress distribution in the diaphysis of the bovine femur. 
In general, residual stress is generated in a material by the indeterminate structure. A simple model of two cylinders of different lengths, a longer cylinder inside the other, will be discussed. First, the inner cylinder is compressed until its length is identical to that of the outer cylinder. Then, the outer cylinder in a nondeformed state is bonded with the inner cylinder under the compressed stress state. When any external loadings are removed, tensile residual stresses remain in the outer cylinder and compressive stresses remain in the inner cylinder, satisfying the equilibrium of forces. This would explain the observed residual stress distribution in the diaphyses of bovine femurs. Research has established that growth of new bone tissue occurs around the cortical surface of the diaphysis (e.g., Currey, 2002). In vivo mechanical loading from body weight increases with growth. Because new tissue develops in a nondeformed state under in vivo loading, tensile stresses may occur in the new surface growth and compressive stresses may remain in the older, deeper bone tissue even after any external loadings have been removed. Based on this hypothesis, although the young bones develop at a rapid pace, the large residual stresses may not be generated in the young bones. This is because the body weight difference during bone formation between the 
cortical surface and deeper regions of the diaphysis in a young bovine may be much smaller than that of a mature bovine.

Bone growth in the diaphysis occurs with shifting of the periosteum enveloping the outer surface of cortical bone. McBride et al. (2011) noted that the periosteum of the adult ovine femoral diaphysis had anisotropic mechanical properties and was pre-stressed in the axial direction. Maturation of the mechanical properties and accompanying changes in the function of the periosteum could also help to explain the findings of the current study.

Because bone tissue has a hierarchical structure-spanning from the macrostructure at the whole-bone level, to the microstructures of osteons and the nanostructures of HAp crystals and collagen fibrils - each structural level must be considered. Previous study has suggested that the residual stresses would be related to the osteon structures and/or their collagen/lamellar/crystallite organization (Yamada et al., 2011a). Furthermore, it is well known that the mechanical and physicochemical properties of bone tissue depend substantially on age (e.g., Raghavan et al., 2012; Dong et al., 2011; Akkus et al., 2004; Rho et al., 2002). As suggested in Table 1, the bovine 
femoral diaphyses expanded centrifugally. The mean tissue age in the surface region may be different in the deeper regions. This would generate the regional differences in mineral crystal characteristics. Further, the strain energy density due to external bending and torsion is maximized at the mid-diaphyseal surface. This can be associated with the regional histological differences (e.g., the distribution of porosity and/or osteons), which would become more obvious with maturation (Skedros et al., 2001; Skedros et al., 2003). Such heterogeneity among these properties may produce a locally indeterminate structure in bone tissue. A long-term study is needed to better characterize the generation of residual stress in bone tissue throughout the hierarchical structure during maturation and ageing.

This study investigated the residual stress distribution in both young and mature bones for the first time and discussed the effects of growth on the residual stress. The results of the study will be of importance in elucidating the mechanism and biomechanical implications of residual stress in bone tissue.

\section{Acknowledgement}


The authors would like to thank Prof. Masahiro Todoh (Hokkaido University),

Prof. Kazuhiro Fujisaki (Hirosaki University), and Dr. Kentaro Kajiwara (JASRI) for scientific and technical support. This work was supported by Grant-in-Aid for Scientific Research (A), MEXT (No. 24240068) and Grant-in-Aid for JSPS Fellows (No. 09J00736). These experiments were performed at the BL28B2 beamline of the SPring- 8 facility with the approval of JASRI (Nos. 2010A1592, 2010B1674, 2011A1783).

\section{Conflict of Interest Statement}

No actual or potential conflicts of interest exist.

\section{References}

Akhtar, R., Daymond, M.R., Almer, J.D., Mummery, P.M., 2011. Lattice strains and load partitioning in bovine trabecular bone. Acta Biomaterialia 7, 716-723.

Akkus, O., Adar, F., Schaffler, M.B., 2004. Age-related changes in physicochemical properties of mineral crystals are related to impaired mechanical function of cortical 
bone. Bone 34, 443-453.

Al Nazer, R., Lanovaz, J., Kawalilak, C., Johnston, J.D., Kontulainen, S., 2012. Direct in vivo strain measurements in human bone - A systematic literature review. Journal of Biomechanics 45, 27-40.

Almer, J.D., Stock, S.R., 2005. Internal strains and stresses measured in cortical bone via high-energy X-ray diffraction. Journal of Structural Biology 152, 14-27.

Almer, J.D., Stock, S.R., 2007. Micromechanical response of mineral and collagen phases in bone. Journal of Structural Biology 157, 365-370.

Currey, J.D., 2002. Bones: Structure and Mechanics. Princeton University Press, USA.

Dong, X.N., Qin, A., Xu, J., Wang, X., 2011. In situ accumulation of advanced glycation endproducts (AGEs) in bone matrix and its correlation with osteoclastic bone 
resorption. Bone 49, 174-183.

Fujisaki, K., Tadano, S., Sasaki, N., 2006. A method on strain measurement of HAP in cortical bone from diffusive profile of X-ray diffraction. Journal of Biomechanics 39, $579-586$.

Fujisaki, K., Tadano, S., 2007. Relationship between bone tissue strain and lattice strain of HAp crystals in bovine cortical bone under tensile loading. Journal of Biomechanics 40, $1832-1838$.

Giri, B., Almer, J.D., Dong, X.N., Wang, X., 2012. In situ mechanical behavior of mineral crystals in human cortical bone under compressive load using synchrotron X-ray scattering techniques. Journal of the Mechanical Behavior of Biomedical Materials 14, 101-112.

Giri, B., Tadano, S., Fujisaki, K., Todoh, M., 2008. Understanding site-specific residual 
strain and architecture in bovine cortical bone. Journal of Biomechanics 41, 3107-3115.

Gupta, H.S., Seto, J., Wagermaier, W., Zaslansky, P., Boesecke, P., Fratzl, P., 2006.

Cooperative deformation of mineral and collagen in bone at the nanoscale. Proceedings

of the National Academy of Sciences 103, 17741-17746.

McBride, S.H., Evans, S.F., Knothe Tate, M.L., 2011. Anisotropic mechanical properties of ovine femoral periosteum and the effects of cryopreservation. Journal of Biomechanics 44, 1954-1959.

Raghavan, M., Sahar, N.D., Kohn, D.H., Morris, M.D., 2012. Age-specific profiles of tissue-level composition and mechanical properties in murine cortical bone. Bone 50, 942-953.

Rho, J.Y., Zioupos, P., Currey, J.D., Pharr, G.M., 2002. Microstructural elasticity and regional heterogeneity in human femoral bone of various ages examined by 
nano-indentation. Journal of Biomechanics 35, 189-198.

Salai, M., Brosh, T., Keller, N., Perelman, M., Dudkiewitz, I., 2000. The effects of prolonged cryopreservation on the biomechanical properties of bone allografts: a microbiological, histological and mechanical study. Cell and Tissue Banking 1, 69-73.

Skedros, J.G., Mason, M.W., Bloebaum, R.D., 2001. Modeling and remodeling in a developing artiodactyl calcaneus: a model for evaluating Frost's Mechanostat hypothesis and its corollaries. The Anatomical Record 263, 167-185.

Skedros, J.G., Sybrowsky, C.L., Parry, T.R., Bloebaum, R.D., 2003. Regional differences in cortical bone organization and microdamage prevalence in Rocky Mountain mule deer. The Anatomical Record 274A, 837-850.

Tadano, S., Giri, B., Sato, T., Fujisaki, K., Todoh, M., 2008. Estimating nanoscale deformation in bone by X-ray diffraction imaging method. Journal of Biomechanics 41, 
945-952.

Tadano, S., Giri, B., 2011. X-ray diffraction as a promising tool to characterize bone nanocomposites. Science and Technology of Advanced Materials 12, 064708.

Tadano, S., Okoshi, T., 2006. Residual stress in bone structure and tissue of rabbit's tibiofibula. Bio-Medical Materials and Engineering 16, 11-21.

Yamada, S., Tadano, S., 2010. Residual stress around the cortical surface in bovine femoral diaphysis. Journal of Biomechanical Engineering 132, 044503.

Yamada, S., Tadano, S., Fujisaki, K., 2011a. Residual stress distribution in rabbit limb bones. Journal of Biomechanics 44, 1285-1290.

Yamada, S., Tadano, S., Todoh, M., Fujisaki, K., 2011b. Residual stress distribution in the bovine femoral diaphysis measured by synchrotron. Journal of Biomechanical 
Science and Engineering 6, 114-124.

Yamada, S., Tadano, S., Fujisaki, K., Kodaki, Y., 2013. Influence of osteon area fraction and degree of orientation of HAp crystals on mechanical properties in bovine femur. Journal of Biomechanics 46, 31-35. 


\section{Figure and table legends}

Figure 1 Coordinate system and relationship between interplanar spacing $d$ of HAp crystals and inclination angle $\psi$. The coordinate system is fixed at each measurement location in the cortical bone. The lattice planes of HAp crystals with orientation $\psi$ diffract synchrotron white X-rays and the interplanar spacing $d_{\psi}$ are measured using Bragg's law, the fundamental equation of X-ray diffraction. Although the interplanar spacing $d_{\psi}$ is constant in the nonstrained state, the lattice planes deform under tensile residual stress to the bone axis. Interplanar spacing $d_{1}$ is largest in lattice planes oriented in the stress direction $\left(\psi_{1}=90^{\circ}\right)$ and smallest in those oriented normally to the surface $\left(d_{1}>d_{2}>d_{3}\right)$. The relationship between $d$ and $\psi$ is affected by the magnitude of the stress.

Figure 2 Diaphysis specimens cut from bovine femurs. Measurements were conducted at the center of the specimens, corresponding to the center of the femur. The diaphysis specimens from Group Y were measured at $0.5-\mathrm{mm}$ intervals from the outer surface to 
the inner region at four locations: anterior, posterior, lateral, and medial. The Group $\mathrm{M}$ specimens were measured at 1-mm intervals at the four locations. The study defined anterior as the head side and posterior as the tail side of the animal when the limbs were extended.

Figure 3 (a) Measurement setup for X-ray diffraction at the BL28B2 beamline of SPring-8. The specimen was fixed on a swivel stage to enable changes in the angle $\psi$ $\left(90.0^{\circ}, 71.6^{\circ}, 63.4^{\circ}\right.$, and $\left.56.8^{\circ}\right)$. The specimen was irradiated with the X-ray beam at an incident angle $\theta$ of $2.5^{\circ}$ and the diffraction angle $2 \theta$ fixed at $5^{\circ}$. The diffracted X-rays were measured using a solid-state detector (SSD). The synchrotron white X-ray beam was collimated with a slit (Slit 1), and scattered X-rays were eliminated with two slits (Slits 2 and 3). (b) The region measured for residual stress in each location was a parallelepiped (approximately $5500 \times 250 \times 240 \mu \mathrm{m}$ ). The longitudinal direction of the region was aligned along the circumferential direction of the diaphysis specimen.

Figure 4 Typical X-ray diffraction profile of a diaphysis specimen. The horizontal axis 
indicates the energy of diffracted X-rays. The peak of the (002) plane in the HAp crystals is sharp with a distinctly high intensity. The peak position was detected between 39.8 and $43.1 \mathrm{keV}$.

Figure 5 Typical cross-sectional shapes and measured regions of the diaphysis specimens in (a) Group Y and (b) Group M. These images were observed by cutting the specimens after measurement. Triangular marks in the image indicate the measurement locations: anterior (A), posterior (P), lateral (L), and medial (M). Dark line marks indicate the measured regions of the diaphysis specimens.

Figure 6 Typical radial distribution of residual stress in the bone axis from the outer surface to the deeper region of the diaphysis specimens from (a) Group Y and (b) Group $\mathrm{M}$ at the four locations: anterior, posterior, lateral, and medial. The deepest locations indicated in this figure do not correspond to the inner surfaces of the diaphysis specimens. The cortical thickness at each location in the Group Y specimen was $3.6 \mathrm{~mm}$ at anterior, $3.1 \mathrm{~mm}$ at posterior, $2.3 \mathrm{~mm}$ at lateral, and $4.0 \mathrm{~mm}$ at medial. The cortical 
thickness in the Group M specimen was $9.6 \mathrm{~mm}$ at anterior, $7.8 \mathrm{~mm}$ at posterior, 7.1

$\mathrm{mm}$ at lateral, and $9.3 \mathrm{~mm}$ at medial.

Figure 7 Residual stresses between the two groups at the (a) anterior and (b) posterior positions. Each value indicates the average of five specimens, and the errors corresponding to their standard deviations.

Figure 8 Comparisons of residual stresses between the surface and deeper regions of the diaphysis specimens and the averages of cross-sectional area. The inner region in Group M is located at the 3-mm depth from the surface, and that in Group $\mathrm{Y}$ is located at the $1.5-\mathrm{mm}$ depth. Each bar of residual stresses indicates the average of the four positions in five specimens $(n=20)$. The bar $(*)$ indicates the average of 18 measurements and the bar $(* *)$ indicates the average of 17 measurements after excluding some unavailable data.

Figure 9 Relationship between the residual stresses at the surface region of the 
diaphysis specimens and the cortical thickness at the measured locations.

Table 1 Average of specimen size at the measurement position of the diaphysis specimens in each group. 
Figures and tables

Figure 1

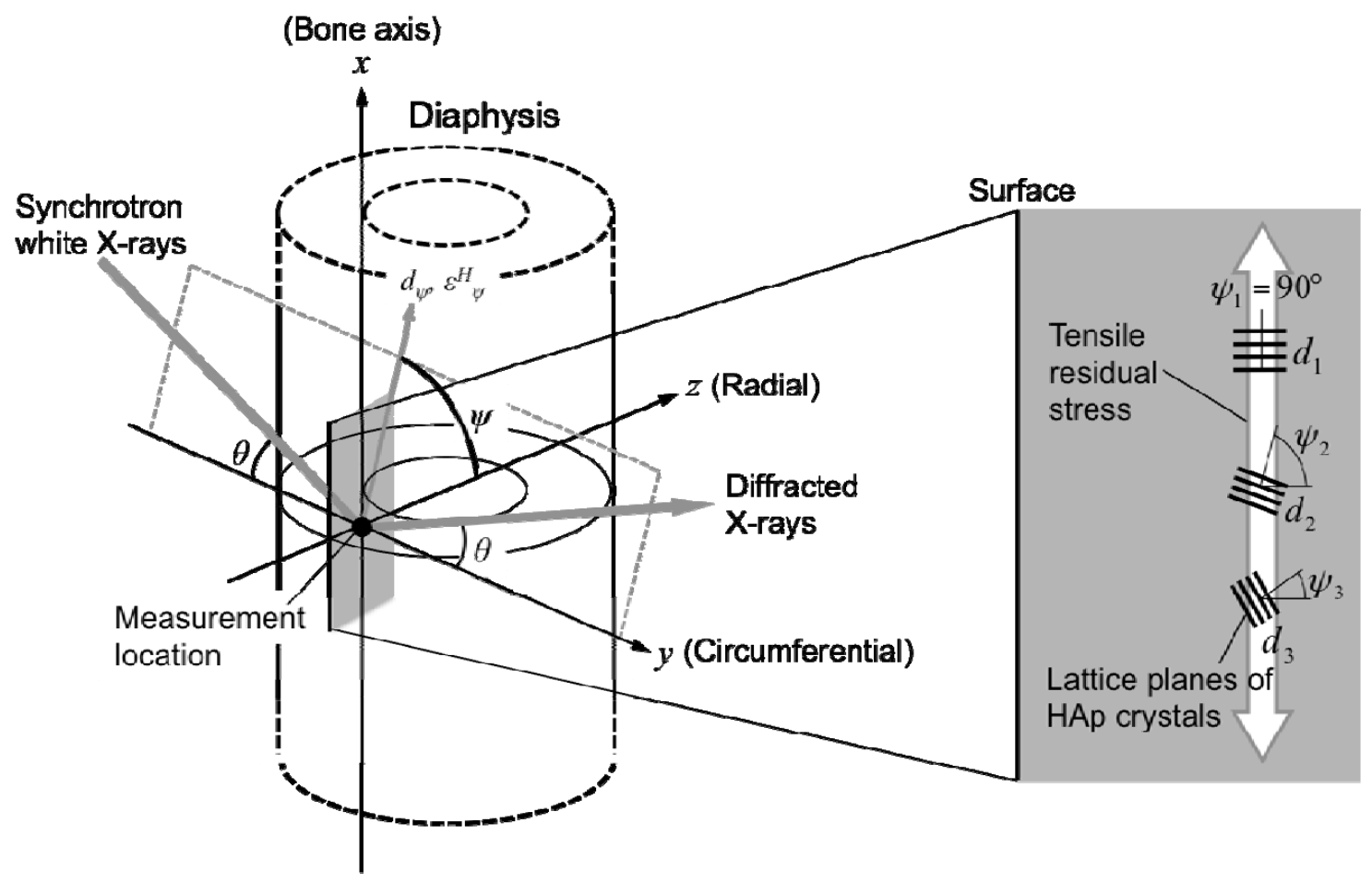


Figure 2

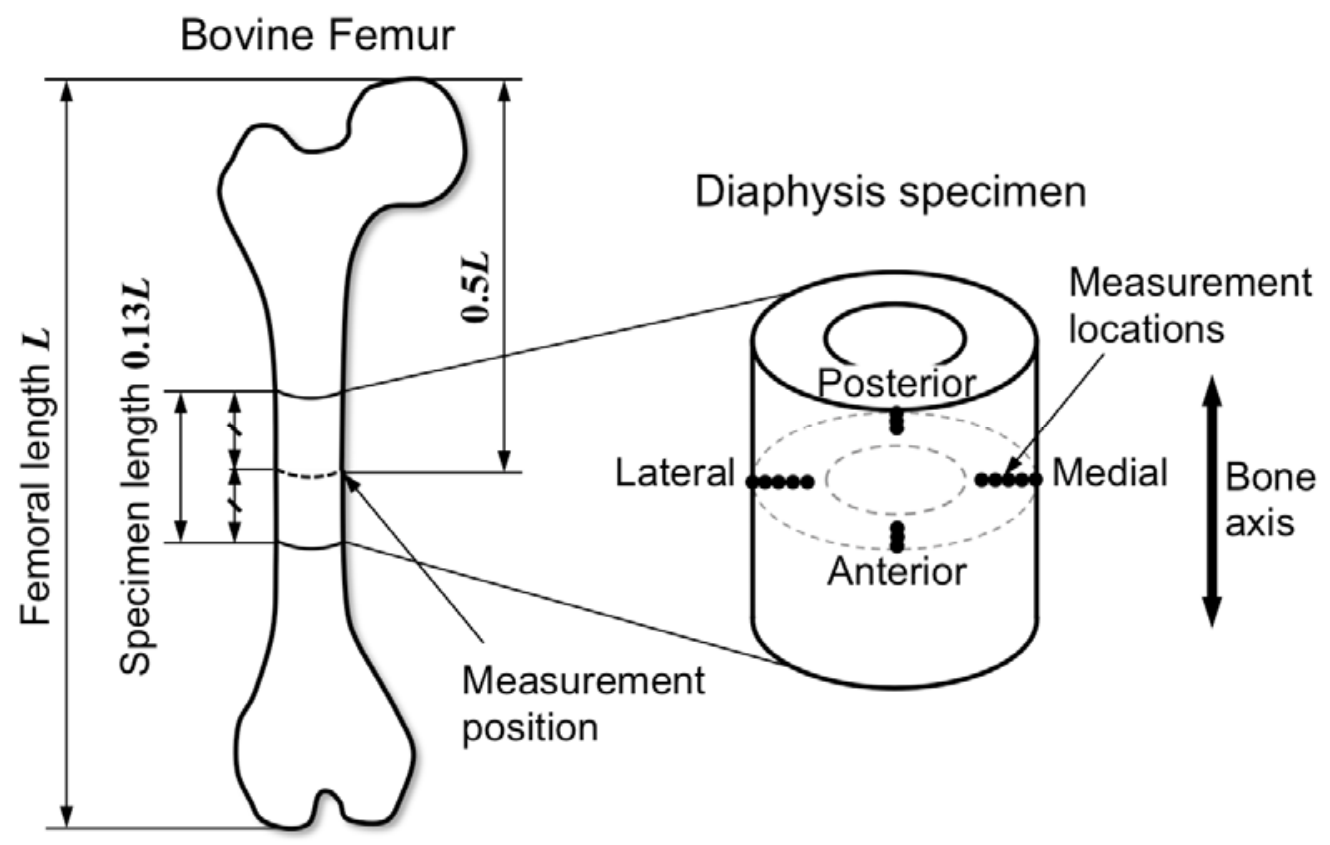


Figure 3

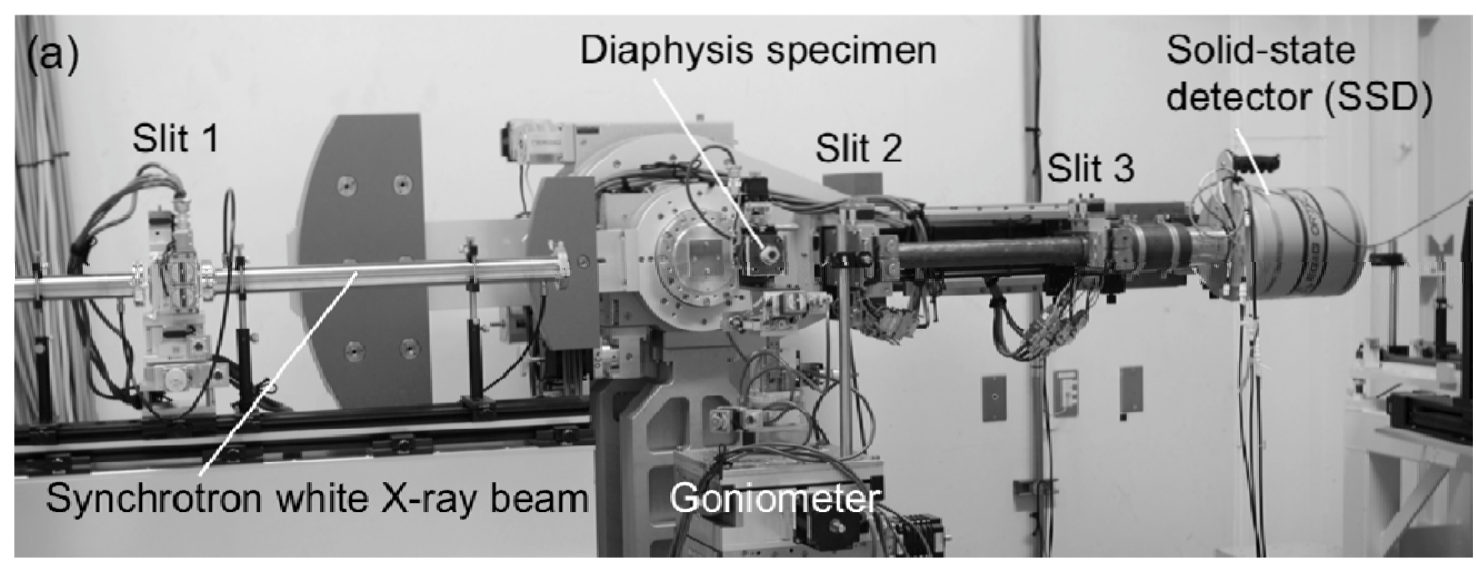

(b)

Rotation of the specimen

to change the angle $\psi$

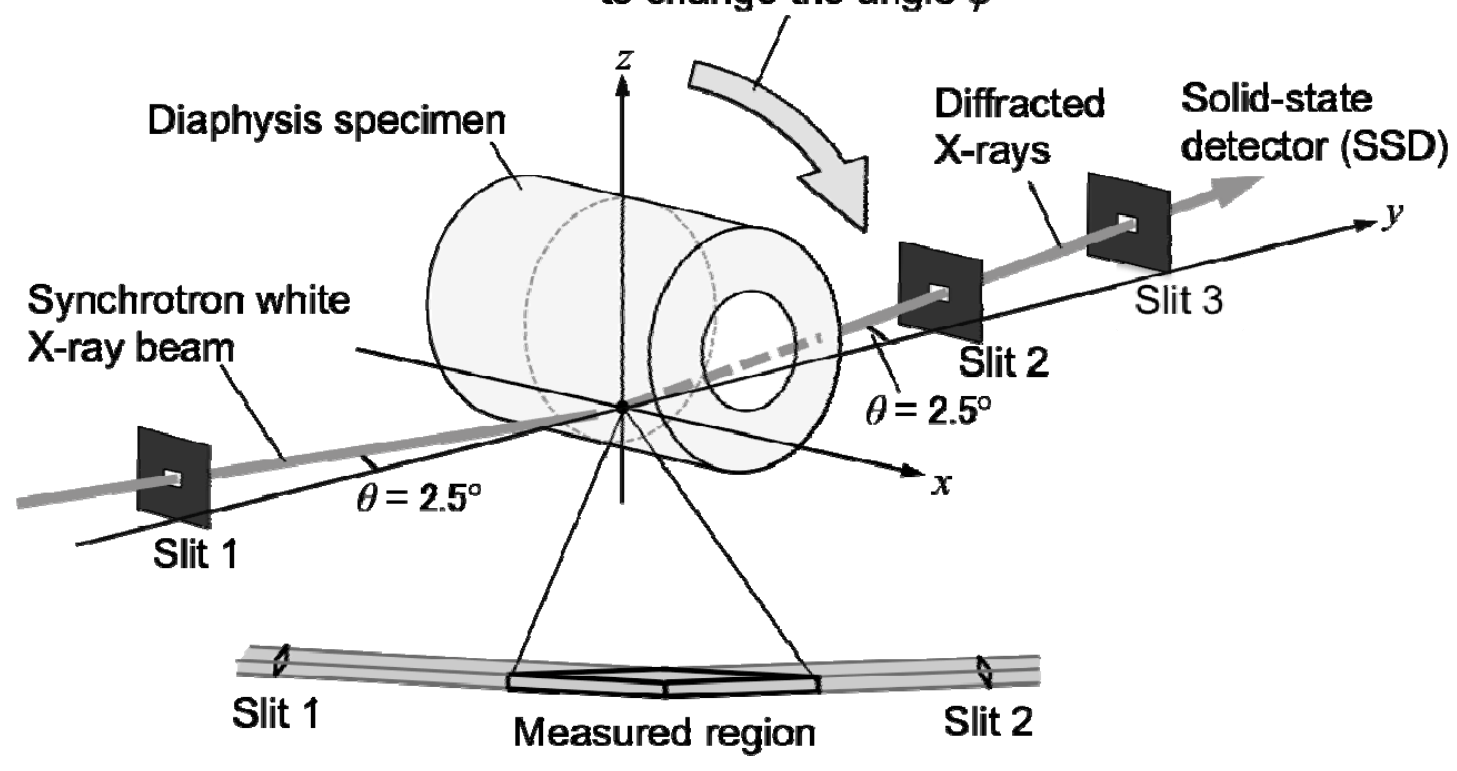


Figure 4

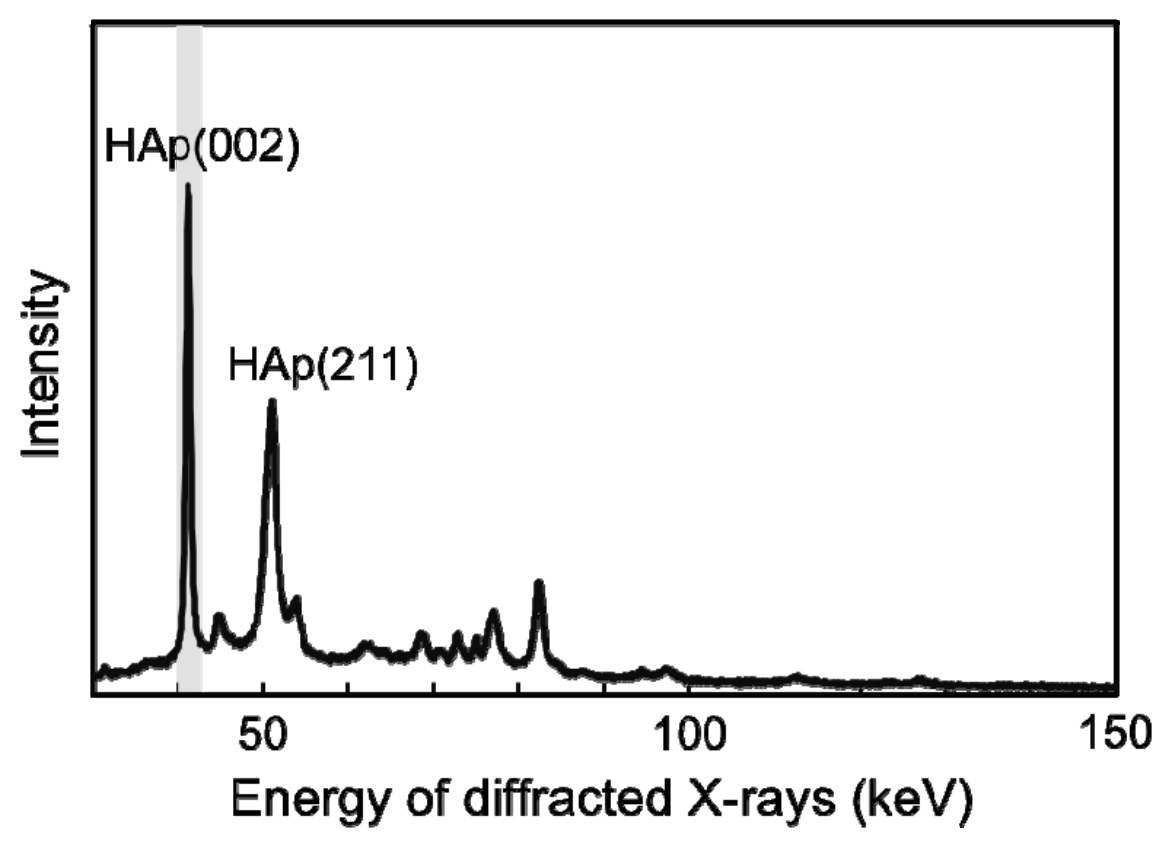


Figure 5

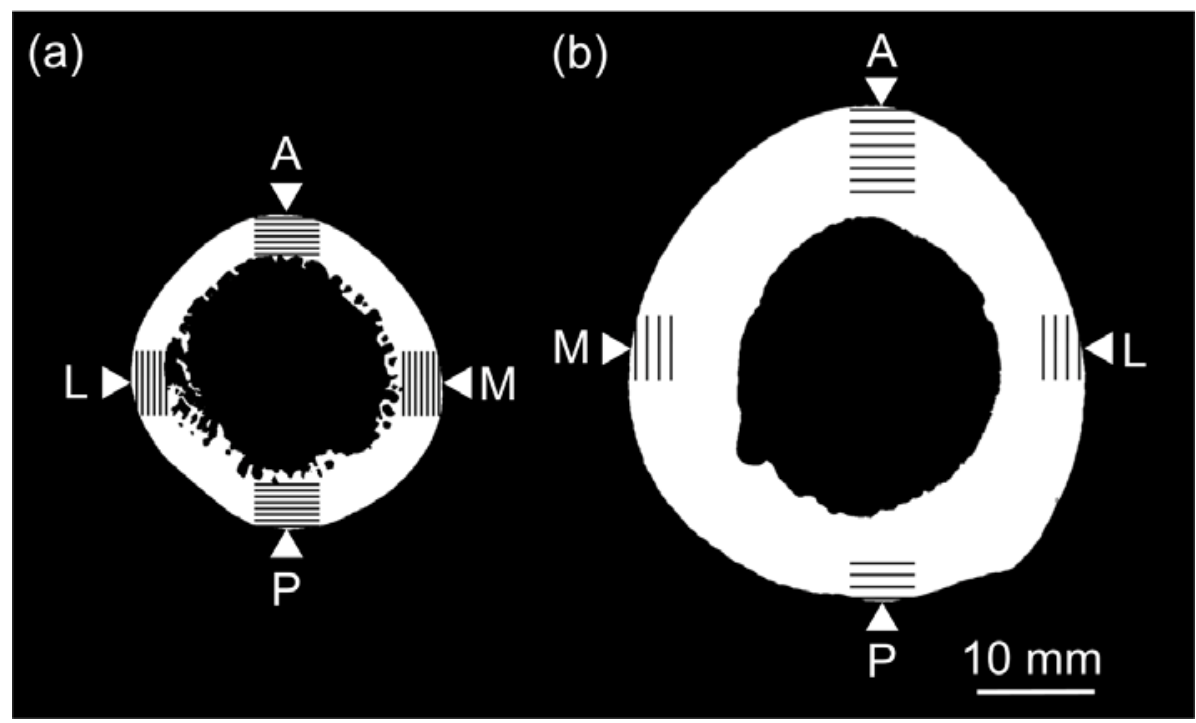


Figure 6

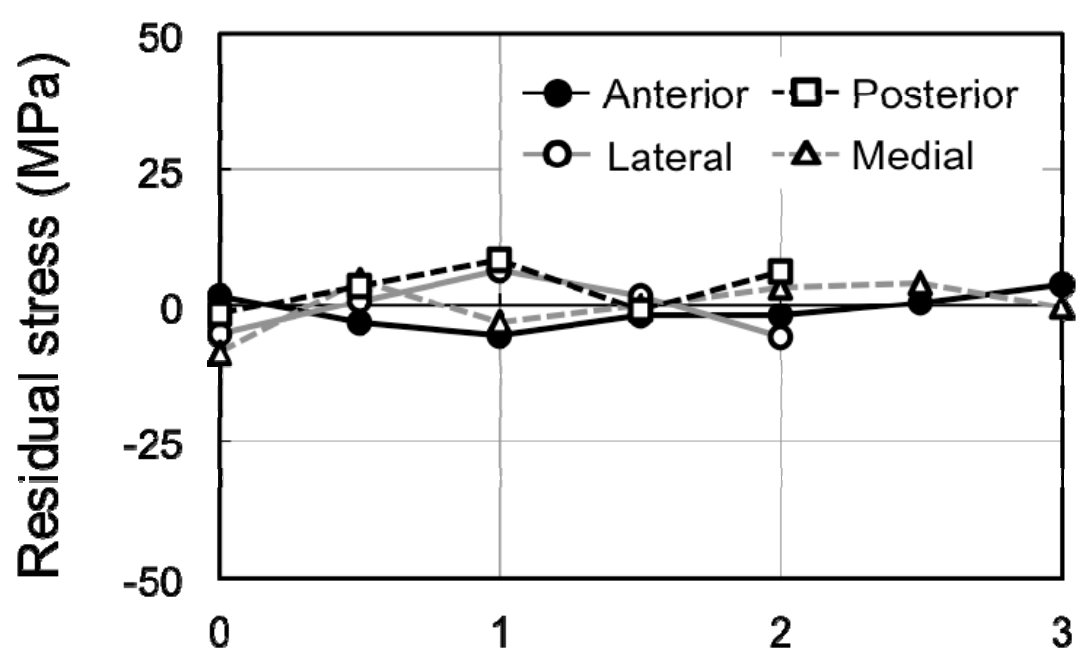

Depth from the specimen surface $(\mathrm{mm})$

(a) Group $Y$

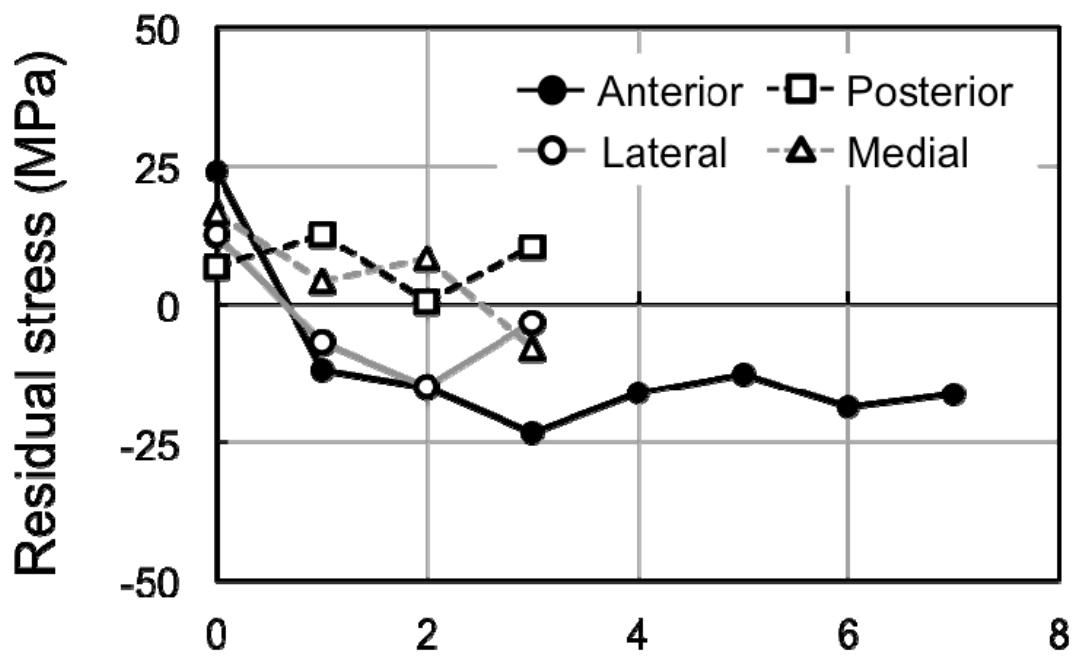

Depth from the specimen surface $(\mathrm{mm})$

(b) Group M 
Figure 7

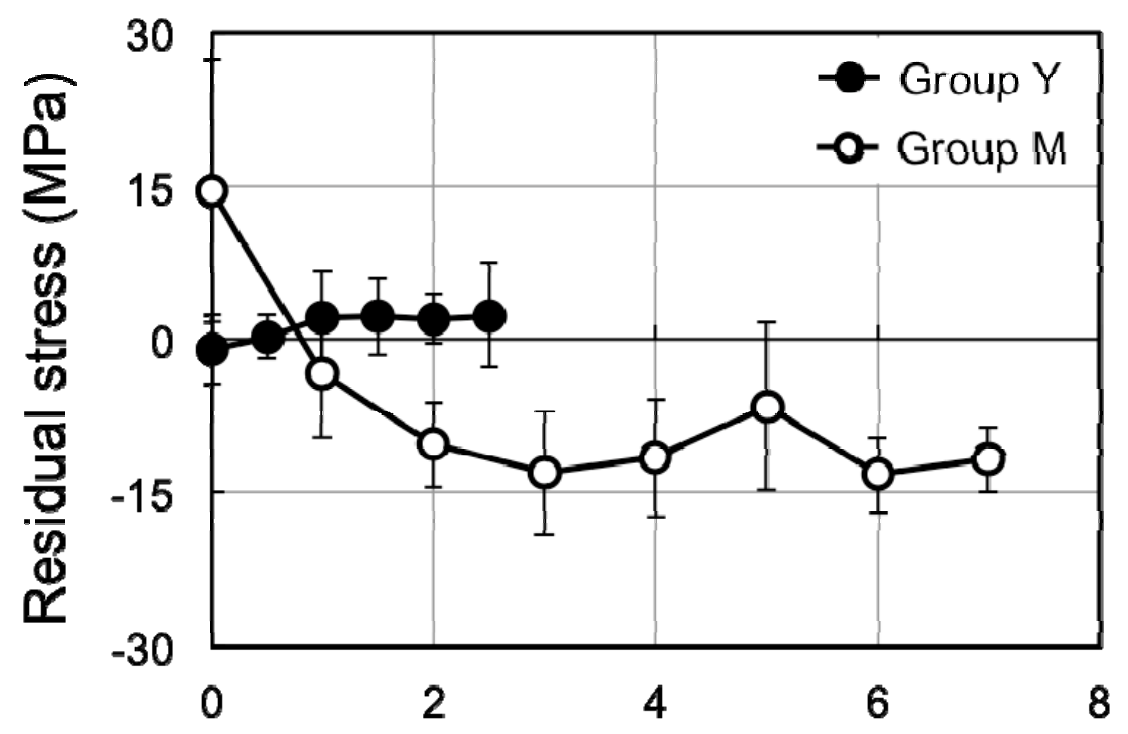

Depth from the specimen surface $(\mathrm{mm})$

(a) Anterior

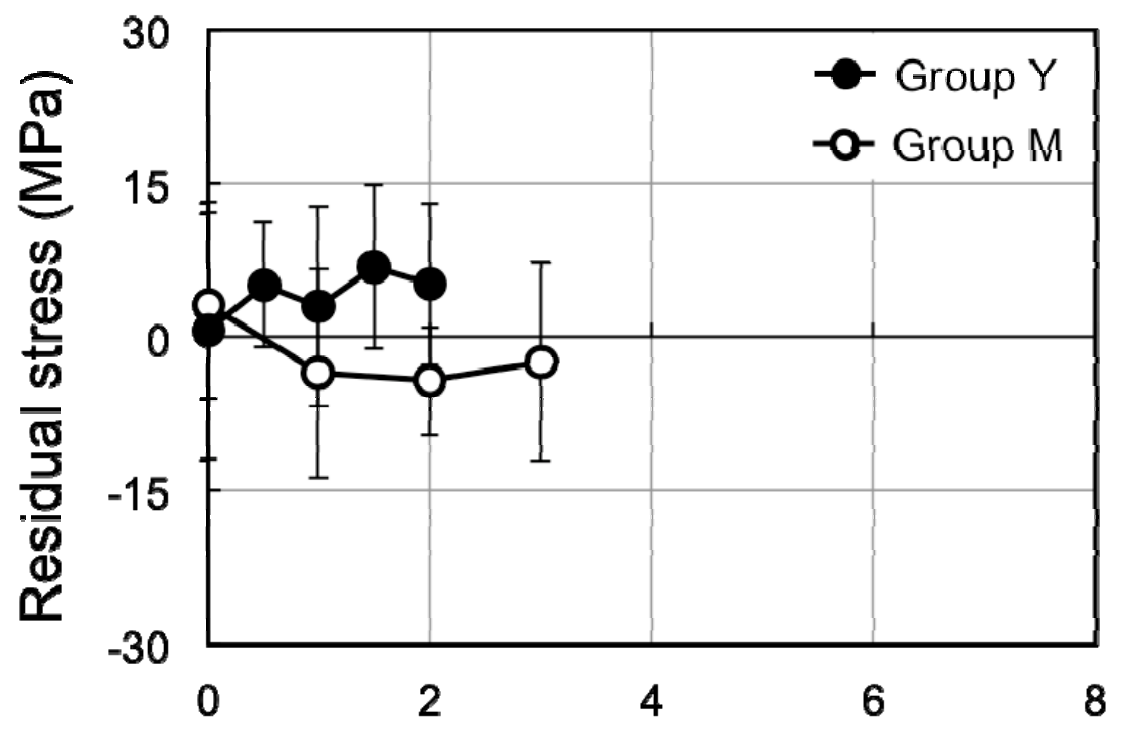

Depth from the specimen surface $(\mathrm{mm})$

(b) Posterior 
Figure 8

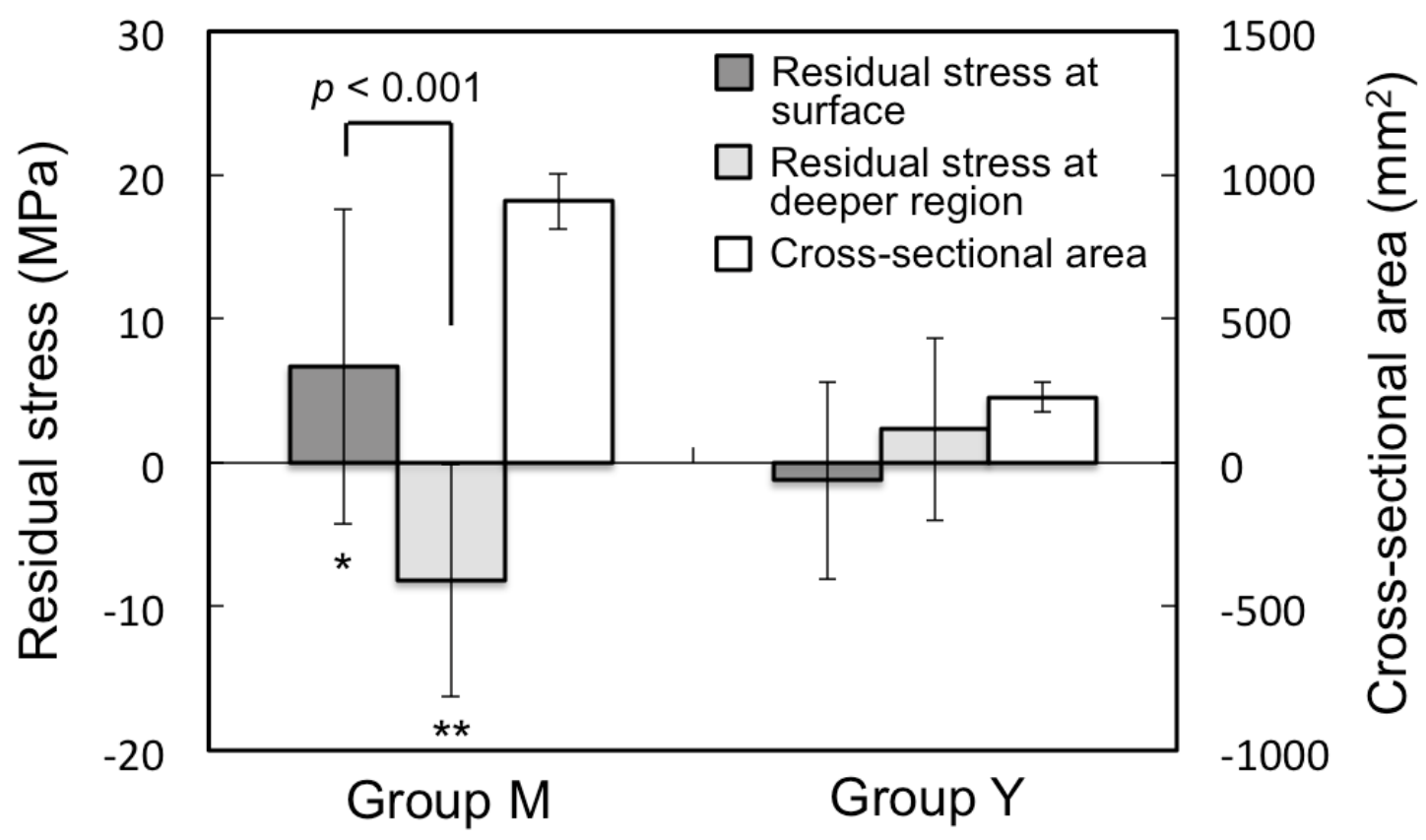


Figure 9

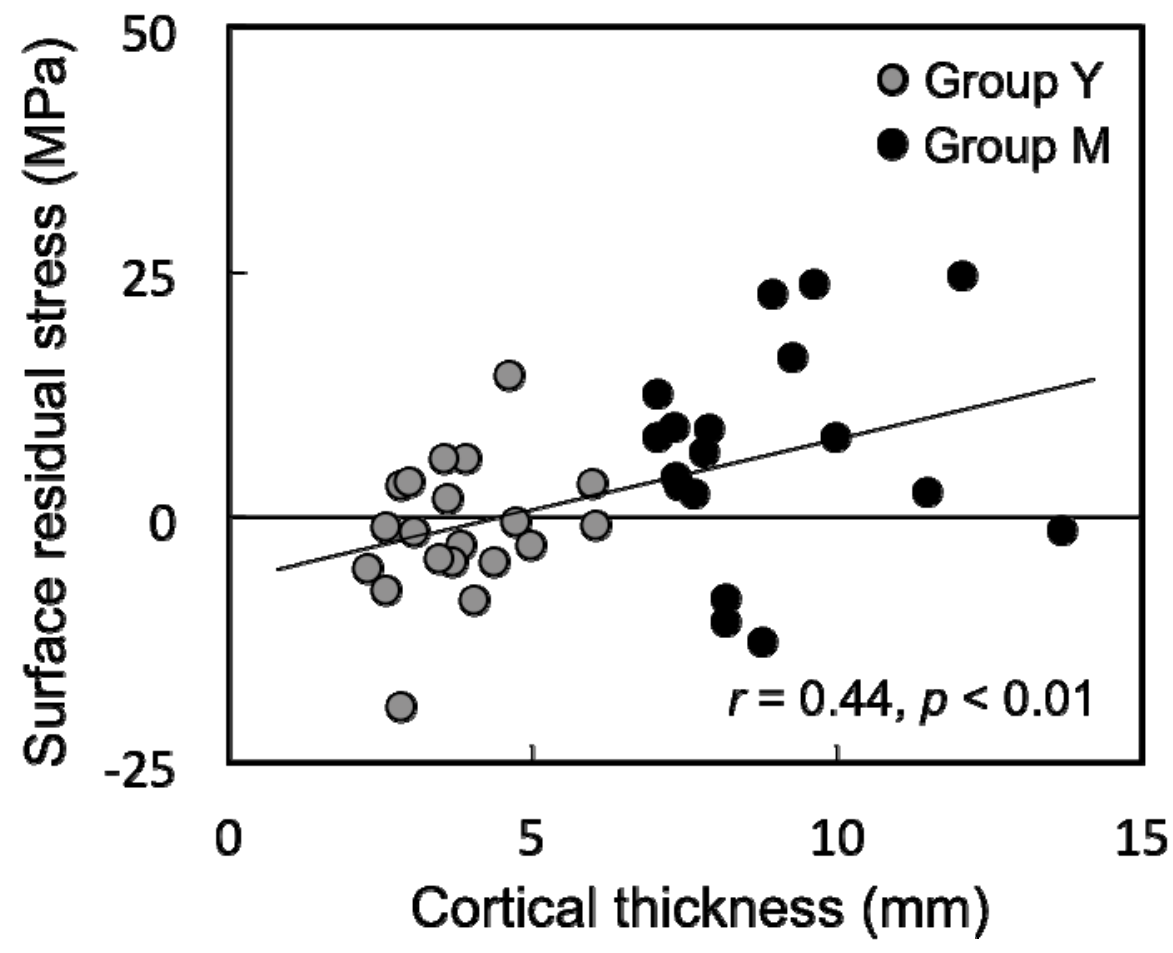


Table 1

\begin{tabular}{lcccccc}
\hline \multirow{2}{*}{ Specimen size and shape } & & \multicolumn{2}{c}{ Group Y } & & \multicolumn{2}{c}{ Group M } \\
\cline { 3 - 4 } \cline { 6 - 7 } & & Mean & S.D. & & Mean & S.D. \\
\hline Cross-sectional area & $\left(\mathrm{mm}^{2}\right)$ & 228 & 51 & & 910 & 97 \\
Cortical thickness & & & & & & \\
$\quad$ Anterior & $(\mathrm{mm})$ & 3.8 & 0.7 & & 11.2 & 1.7 \\
$\quad$ Posterior & $(\mathrm{mm})$ & 4.1 & 1.1 & & 7.8 & 0.5 \\
Lateral & $(\mathrm{mm})$ & 3.0 & 0.9 & & 7.8 & 0.8 \\
$\quad$ Medial & $(\mathrm{mm})$ & 4.3 & 0.9 & & 9.1 & 1.1 \\
$\quad$ Averages of four positions & $(\mathrm{mm})$ & 3.8 & 0.9 & & 9.0 & 0.9 \\
Diaphyseal width & & & & & & \\
$\quad$ Anterior-Posterior & $(\mathrm{mm})$ & 23.4 & 2.0 & & 44.8 & 1.9 \\
$\quad$ Lateral-Medial & $(\mathrm{mm})$ & 23.9 & 1.5 & & 40.1 & 1.6 \\
\hline
\end{tabular}

